\title{
À la mémoire d'Anne-Marie Grignon
}

Professeur au département d'Études françaises

de l'Université du Nouveau-Brunswick

Anne-Marie Grignon est morte le 7 novembre 1988. Sa disparition nous consterne tous. J'aimerais rappeler ici les étapes de sa trop brève carrière en linguistique.

Pendant ses études a l'Université de Montréal, elle se spécialise en phonologie du japonais - une langue dont elle a une grande maitrise. Étudiante brillante, ses efforts sont reconnus par les organismes gouvernementaux du Québec et du Canada qui lui accordent des bourses pour sa maitrise, son doctorat et ses recherches post-doctorales. Elle obtient sa maîtrise en 1980 puis son doctorat en 1984. Elle mène ses recherches post-doctorales sur la théorie de la morphologie et l'aphasie à l'Université McGill et au Centre hospitalier de la Côte-des-Neiges. Elle commence sa carrière de professeur en 1985 au département d'Études françaises de l'Université du Nouveau-Brunswick à Fredericton. Son activité professorale porte sur l'enseignement du français comme langue seconde et langue maternelle et sur la linguistique. Elle participe activement aux tâches administratives en ouvrant dans de nombreux comités de la Faculté des arts et du Département.

Elle poursuit et fait connaître ses recherches sur la structure phonologique et morphologique du japonais. Elle fait partie du jury de sélection du dernier congrès NELS à Toronto en 1987. Elle publie "On the Structure of the Japanese Rime", McGill Working Papers in Linguistics 2:2, 1985; "The 'h/p,b' Alternation", Journal of Asian Culture 9, 1985; "Japanese Complex Predicates and Lexical Phonology", Papers in Japanese Linguistics 12, 1987. Elle commençait tout juste avec quelques étudiants de nouvelles recherches sur la morphologie du français dont elle présentait certains résultats "The Suffixes - age and -ment in Modern French: Dictionary vs. Speakers" au dernier congrès de LACUS à East Lansing (Michigan) et "Grammaire interne, norme externe et norme intériorisée" au dernier congrès de la SILF à Moncton.

Tous se rappelleront cette femme dynamique, enjouee, souriante, et d'une immense générosité.

Yves-Charles Morin

Universitẻ de Montréal 


\title{
Editor's Acknowledgements
}

The editor would like to thank the following persons for having served as referees for articles during the year 1988:

\author{
Joseph Aoun \\ Jean-Marc Authier \\ Bruce Bagemihl \\ Denis Bouchard \\ Rose-Marie Dechaine \\ John Dunn \\ Dale Kinkade \\ Victor Manfredi \\ Karen Michelson \\ Yves-Charles Morin \\ Carole Paradis \\ Marisa Rivero \\ Yves Roberges \\ Michael Rochemont \\ Bernard Rochet \\ Doug Walker \\ Linda Walsh \\ Hanni Woodbury
}

